\title{
Novas combinações para espécies brasileiras de Acanthaceae
}

\author{
New combinations to Brazilian species of Acanthaceae
}

\author{
Sheila R. Profice ${ }^{1}$
}

\begin{abstract}
Resumo
Durante a preparação da lista das espécies de Acanthaceae para a Lista de Espécies da Flora do Brasil foi detectada a necessidade da proposição de novas combinações, novos sinônimos, novos nomes e esclarecimentos a cerca da delimitação de algumas espécies. Tais novidades nomenclaturais são aqui apresentadas. Palavras-chave: Acanthaceae, Justicia, Ruellia, nomenclatura.
\end{abstract}

\begin{abstract}
During the preparation of the list of species of Acanthaceae for the List of Species of the Brazilian Flora the necessity of present new combinations, new synonyms, new names, and taxonomic notes about the delimitation of some species was observed. These nomenclatural novelties are here presented.

Key words: Acanthaceae, Justicia, Ruellia, nomenclature.
\end{abstract}

\section{Introdução}

A monografia de Nees von Esenbeck (1847) ainda é a única obra que trata as espécies brasileiras da família Acanthaceae como um todo. Neste estudo, a falta de consistência dos caracteres diagnósticos adotados pelo autor na circunscrição dos gêneros levou autores posteriores a propor novos conceitos genéricos e, em muitos casos, a sinonímia de diversos gêneros.

Entre os vários autores que publicaram novas espécies desta família para a Flora do Brasil destacamse Lindau (1895a, 1898) e Rizzini (1946, 1949, 1950, 1952, 1956). Atualmente, os conceitos genéricos estabelecidos por estes autores são amplamente questionados e as espécies descritas por eles necessitam de atualizações taxonômicas. Neste contexto foram propostas mudanças taxonômicas em espécies da América do Sul, incluindo Brasil, dos gêneros Justicia L. (Ezcurra 2002) e Ruellia L. (Ezcurra 1993). Apesar de várias espécies descritas por Nees von Esenbeck, Lindau e Rizzini em gêneros considerados sinônimos de outros em trabalhos recentes de flora e revisões, vários desses táxons ainda necessitam ser transferidos para gêneros atualmente aceitos em Acanthaceae.
O presente estudo tem por objetivo de apresentar as novidades nomenclaturais em Acanthaceae tendo em vista a divulgação para a publicação da Lista de Espécies da Flora do Brasil.

\section{Material e Métodos}

Para análise das espécies de Justicia, gênero de maior complexidade taxonômica, considera-se Justicia sensu Graham (1988) e aceita-se Ruellia sensu lato. Este estudo tem por base o material botânico depositado no Herbário do Jardim Botânico do Rio de Janeiro (RB) e cedido por empréstimo, incluindo fotografias dos tipos, dos seguintes herbários citados pela sua respectiva sigla, conforme Holmgren et al. (1990): BM, C, F, G, GZU, $\mathrm{K}, \mathrm{NY}, \mathrm{Pe}$ W.

\section{Resultados}

1. Justicia araucariensis Profice, nom. nov. para Chaetothtylax vestitus Rizzini, Dusenia 3(3): 191. 1952. Tipo: BRASIL. PARANÁ: Parque Nacional Iguaçu, A.P. Duarte \& E. Pereira 1927 (holótipo RB!).

Ezcurra (2002) sem examinar o material-tipo de Chaetothtylax vestitus, depositado no herbário $\mathrm{RB}$, inclui esta espécie na sinonímia de Justicia 
goudotii V.A.W.Graham, provavelmente pela semelhança na forma da corola. Entretanto, $C$. vestitus difere de J. goudotii (sensu Ezcurra 2002) pela bráctea de oblonga a ovado-lanceolada, com 12-15 mm comprimento ( $v s$. lanceolada, com 4-6 mm compr.), bractéola subulada, com ca. $10 \mathrm{~mm}$ comprimento, revestida por indumento alvo-viloso ( $v s$. lanceolada, com comprimento semelhante ao da bráctea, ciliada) e corola com ca. $3,5 \mathrm{~cm}$ comprimento ( $v s .1,5-2 \mathrm{~cm}$ compr.). Posteriormente, Ezcurra \& Kameyama (2008), no Catálogo Del Cone Sur, colocaram C. vestitus na sinonímia de J. ramulosa (Morong.) Ezcurra, espécies estreitamente relacionadas. Contudo, $C$. vestitus é distinta de J. ramulosa pela bráctea oblonga a ovado-lanceolada (vs. elíptica a obovada). Como o epiteto vestita já está ocupado em Justicia (J. vestita Schultez, 1822) um novo nome é proposto no presente estudo.

2. Justicia bradeana Profice, nom. nov. para Psacadocalymma latifolium Rizzini, Dusenia 3(3): 183. 1952, non Vahl(1791). Tipo: BRASIL. ESPÍRITO SANTO: Vargem Alta, Cachoeiro do Itapemirim, A.C. Brade 19889 (holótipo RB!).

3.Justicia bullata (Nees) Profice, comb. nov. Belloperone bullata Nees in Mart., Fl. bras. 9: 141. 1847. Tipo: BRASIL. Sellow 117, 287 (síntipos B destruídos, fotografia F 8924!).

4. Justicia flosculosa Profice, nom. nov. para Leptostachya laxa Nees in Mart., Fl. bras. 9: 151. 1847, non Anderson (1863). Tipo: BRASIL. BAHIA: Rio Belmonte, Maximilianus Princ. Vidensis s.n. (síntipo W!); Ilhéus, Blanchet 3055 (síntipo G!).

Psacadocalymma falcatum Rizzini, Dusenia 3: 182. 1952. Tipo: BRASIL. ESPÍRITO SANTO: Cachoeira do Itapemirim, A.C. Brade 19903 (holótipo RB!), syn. nov., non Justicia falcata Lamark(1785).

Psacadocalymma falcatum Rizzini var. stenophyllum, Dusenia 3: 182. 1952. Tipo: BRASIL. ESPÍRITO SANTO: Vargem Alta, A.C. Brade 19760 ( holótipo RB!), syn. nov.

Nees von Esenbeck (1847) com base na inflorescência constituída de espigas com pequenas flores, cujo aspecto sugere uma panícula, descreve Leptostachya laxa para a Bahia. Rizzini (1952) utiliza esta mesma característica para descrever para o estado do Espírito Santo Psacadocalymma falcatum e $P$. falcatum var. stenophyllum, diferindo os dois táxons pela forma da folha. Em P. falcatum a folhaé lanceolado- ovada, enquanto na variedade stenophyllum lanceolada. Os materiais-tipo de Leptostachya laxa e Psacadocalymma falcatum são praticamente idênticos. Da mesma forma, o material-tipo de $P$. falcatum var. stenophyllum apresenta considerável similaridade morfológica com os outros materiais-tipo, diferindo destes, apenas pelas folhas mais estreitas e pela quantidade maior de espigas. Assim, propõemse $P$. falcatum e $P$. falcatum var. stenophyllum como sinônimos de Justicia flosculosa.

5. Justicia fulvohirsuta (Rizzini) Profice, comb. nov. Orthotactus fulvohirsutus Rizzini, Dusenia 1: 291. 1950. Tipo: BRASIL. ESPÍRITO SANTO: Jatiboca, Itaguassu, A.C. Brade et al. 18231 (holótipo RB!).

6. Justicia goianiensis Profice, nom. nov. para Thyloglossa glandulosa Nees in Mart., Fl. bras. 9: 117. 1847. Adhatoda glandulosa (Nees) Nees in DC., Prodr. 11: 405. 1847. Tipo: BRASIL. GOIÁS: Rio Tocantins, Pohl s.n. (holótipo W 5007; isótipo GZU!). Justicia glandulosa Pohl, nom. nud.

O nome Justicia glandulosa Roth (Nov. Pl. Sp. p. 13. 1821) é um homônimo anterior, o que não permite a utilização deste. Desse modo, propõe-se o binômio Justicia goianiensis para a espécie brasileira.

7. Justicia itatiaiensis Profice, nom. nov. para Beloperone macrosyphon Rizzini, Rodriguésia 32: 187. 1957. Tipo: BRASIL. RIODE JANEIRO: Itatiaia, Fazenda Val Paraizo, L. Lanstyak 203 (holótipo RB!).

O nome Justicia macrosiphon (Lindau) V.A.W.Graham(Kew Bull.43:605.1988)éumhomônimo anterior de uma espécie da Bolívia, o que não permite a utilização deste para a espécie brasileira.

8. Justicia japurensis Profice, nom. nov. para Sericographis cordata Nees in Mart., Fl. bras. 9: 108. 1847. Tipo: BRASIL. AMAZONAS: Rio Negro, Martius s.n. (holótipo M, fotografia F 20511!).

Sericographis acuminata Nees in Mart., Fl. bras. 9: 109. 1847. Tipo: BRASIL. AMAZONAS: Japurá, Rio Negro, Martius s.n. (holótipo M, fotografia F 20510!).

Nees von Esenbeck (1847) admite que Sericographis acuminata seria uma forma de Sericographis cordata. Os materiais-tipo de Martius s.n. (fotografias F 20510; F 20511) sobre os quais Nees Von Esenbeck descreve as espécies são morfologicamente idênticos e por isso Sericographis cordata constitui em um sinônimo de Justicia japurensis. 
O nome Justicia acuminata (Nees) Lindau (Engler \& Prantl. Natur. Pflanzenfam. 4(3b): 351. 1895), para a espécie do México, e o de J. cordata T.Anderson (J. Proc. Linn. Soc. Bot. 7: 44. 1863), para a espécie africana, são homônimos anteriores, o que não permite a utilização destes para a espécie brasileira.

9. Justicia minensis Profice, nom. nov. para Beloperone lanceolata Mart. ex Nees in Mart., Fl. bras. 9: 141. 1847, non Small (1993). Tipo: BRASIL. MINAS GERAIS: Serra da Piedade, Martius s.n. (síntipo M); Morro do Pilar, Conceição, Sellow 27, 37 (síntipo B destruído, fotografia F 8932!).

10. Justicia monticola (Nees) Profice, comb. nov. Sericographis monticola Nees in Mart., Fl. bras. 9: 111. 1847. Tipo: BRASIL. MINAS GERAIS: Serra do Caraça, Martius s.n. (síntipo M, fotografia F 20513!), Schuch s.n. (síntipo W), Claussen s.n. (síntipo P).

Sericographis hirsuta Nees in Mart., Fl. bras. 9: 112. 1847. Tipo: BRASIL. MINAS GERAIS: Martius s.n. (síntipo M, fotografia F 20512!), Ackermann s.n. (síntipo M); RIO DE JANEIRO: Serra dos Órgãos, Gardner 587 (síntipo K), Schuch 4749 (síntipo W), syn. nov.

Beloperone holochila Rizzini, Rev. Bras. Biol. 6: 524. 1946. Justicia holochila (Rizzini) Profice, Eugeniana 20: 3. 1993. Tipo: BRASIL. RIO DE JANEIRO: Serra dos Órgãos, S. Araujo \& E. Pereira 517 (holótipo RB!), syn. nov.

Nees von Esenbeck (1847) difere Sericographis monticola de S. hirsuta pela folha estreita, hirsuta e com pecíolo de comprimento maior. Rizzini (1946) caracteriza Beloperone holochila pelo habito escandente, pilosidade hirsuta e inflorescência em espiga secundiflora, as mesmas características diagnósticas das espécies descritas por Nees von Esenbeck. Além destas características diagnósticas há outras semelhanças morfológicas entre os materiais-tipo das espécies de Nees von Esenbeck coletadas por Martius s.n. (fotografias F 20512; F 20513) e a coleta de S. Araujo \& E. Pereira 517, tipo da espécie de Rizzini, não havendo motivo para manter S. hirsuta e B. holochila separadas de Justicia monticola.

11. Justicia parabolica (Nees) Profice, comb. nov. Sericographis parabolica Nees in Mart., Fl. bras. 9: 111. 1847. Jacobinia parabolica (Nees) Lindau, in Engler \& Prantl., Natur. Pflanzenfam. 4(3b): 351. 1895. Tipo: BRASIL. SÃO PAULO: Sellow s.n. (holótipo B 209 destruído, fotografia F 8913!).
Sericographis lineolata Rizzini, Arq. Jard. Bot. Rio de Janeiro 9: 60, tab. III, fig.1. 1949. Tipo: BRASIL. ESPIIIRITO SANTO: Castelo, A.C.Brade 19171 (holótipo RB!), syn. nov.

Ezcurra \& Kameyama (2008) colocaram Sericographis parabolica como sinônimo de Justicia brasiliana, espécie com distribuição no sul e sudeste do Brasil e em países limítrofes (Argentina e Paraguai). Justicia brasiliana é uma espécie distinta de J. parabolica, principalmente pela inflorescência em espiga subséssil, axilar, densa. Contudo, o material Brade 19171, sobre o qual Rizzini descreve $S$. lineolata, em nada difere morfologicamente do material-tipo coleta de J. parabolica (Sellow s.n. fotografia F 8913), sendo por este motivo proposta S. lineolata como sinônimo.

12. Justicia pohliana Profice, nom. nov. para Beloperone hirsuta Nees in Mart, Fl. bras. 9: 143. 1847. Tipo: BRASIL. RIO DE JANEIRO: Serra do Mar, Martius s.n (síntipo M); MINAS GERAIS: Ackermann s.n (síntipo M), Camargos, Pohl s.n. (síntipo W 3530!), Sellow s.n. (síntipo B 205 destruído, fotografia F 8931!).

Beloperone hirsuta var. longifolia Nees in Mart, Fl. bras. 9: 143. 1847. Tipo: BRASIL. MINAS GERAIS: Ackermann s.n (síntipo M), Schuch s.n. (síntipo W, fotografia F 32683!), syn. nov.

Nees von Esenbeck (1847) descreve Beloperone hirsuta var. longifolia como distinta da variedade típica pela folha oblonga. Em relação a este caráter em nada difere do material-tipo Pohl s.n. (W 3530), sobre o qual Nees von Esenbeck descreve a variedade típica. Assim, coloca-se $B$. hirsuta var. longifolia na sinonímia de Justicia pohliana. O nome J. hirsuta Jacq. (Enun. Syst. Pl. p. 11. 1760) e J. longifolia Vahl (Symb. Bot. 2: 4. 1791) são homônimos anteriores, o que não permite a utilização destes para a espécie brasileira.

13. Justicia sellowiana Profice, nom. nov. para Beloperone diclipterioides Nees in Mart., Fl. bras. 9: 136. 1847, non Lindau (1894). Tipo: BRASIL. Sellow 39 (holótipo B destruído, fotografia F 8930!).

14. Ruellia helianthema (Nees) Profice, comb. nov. Dipteracanthus helianthemum Nees in Mart., Fl. bras. 9: 36. 1847. Tipo: BRASIL. BAHIA: Monte Santo, Martius s.n. (isossíntipo GZU!); Rio Preto, Pohl s.n. (isossíntipo GZU!). 
15. Ruellia riedeliana Profice, nom. nov. para Arrhostoxylum album Nees in Mart., Fl. bras. 9: 60. 1847. Tipo: BRASIL. ESPÍRITO SANTO: Vitória, Max. Princ. Vidensis s.n (isossíntipo BR!); RIO DE JANEIRO: Macaé, Riedel s.n.(síntipo LE).

O nome Ruellia alba Nees (Mart., Fl. bras. 9: 55. 1847) é homônimo anterior, o que não permite a utilização deste para a espécie brasileira.

16. Ruellia salviifolius (Nees) Profice, comb. nov. Stemonacanthus salviifolius Nees in Mart., Fl. bras. 9: 54. 1847. Tipo: BRASIL. Serra S. Felis, Rio Trahira, Pohl s.n. (holótipo W n.1930!).

17. Ruellia serrana Profice, nom. nov. para Arrhostoxylum glabrum Nees in Mart., Fl. bras. 9: 58, tab. 6. 1847, non Roth (1821). Tipo: BRASIL. Serra do Chumbo, Pohl s.n. (síntipo W n. 3283!); BAHIA: Ilhéus, Blanchet s.n. (síntipos G n.3540, K).

\section{Referências}

Ezcurra, C. 1993. Systematic of Ruellia (Acanthaceae) in Southern South America. Annals of the Missouri Botanical Garden 80: 787-845.

Ezcurra, C. 2002. El género Justicia (Acanthaceae) en Sudamérica Austral. Annals of the Missouri Botanical Garden 89: 225-280.

Ezcurra, C. \& Kameyama, C. 2008. Acanthaceae. In: Catálogo de las plantas vasculares Del Cono Sur
(Argentina, sur de Brasil, Chile, Paraguay y Uruguay) Monographs in Systemtic Botany Missouri Botanical Garden 107: 985-1006.

Graham, V.A.W. 1988. Delimitation and infra-generic classification of Justicia (Acanthaceae). Kew Bulletin 34: 551-624.

Holmgren, P.K.; Holmgren, N.H. \& Barnett, L.G. 1990. Index Herbariorum. The New York Botanical Garden, New York. 693p.

Lindau, G. 1895a. Acanthaceae Americanae. Bulletin Herbier Boissier 3: 361-372.

Lindau, G. 1895b. Acanthaceae. In: Engler, A. \& Prantl, H. (eds.). Die Natürlichen Pflanzenfamilien 4: 274-354.

Lindau, G. 1898. Plantae novae americanae imprimis Glaziovinae II. Acanthaceae. Botanische Jahrbucher fur Systematic 25: 47.

Nees von Esenbeck. 1847. Acanthaceae. In: Martius, K.P.; Eichler, A.G. \& Urban, I. (ed.). Flora brasiliensis. Vol. 9. F. Fleischer, Lipsiae. Pp. 1-164.

Rizzini, C.T. 1946. Aliquit novi Acanthacearum. Revista Brasileira Biologia 6: 521-525.

Rizzini, C.T. 1949. Contribuição ao conhecimento da tribo Justicieae (Acanthaceae). Arquivo Jardim Botânico Rio de Janeiro 9: 38-67.

Rizzini, C.T. 1950. Acanthaceae espiritosantenses novae. Dusenia 1: 291-292.

Rizzini, C.T. 1952. Acanthacearum delectus Brasiliensium. Dusenia 3: 181-196.

Rizzini, C.T. 1956. Notulae de Acanthaceis novis seu minus cognitis. Dusenia 7: 299-305. 